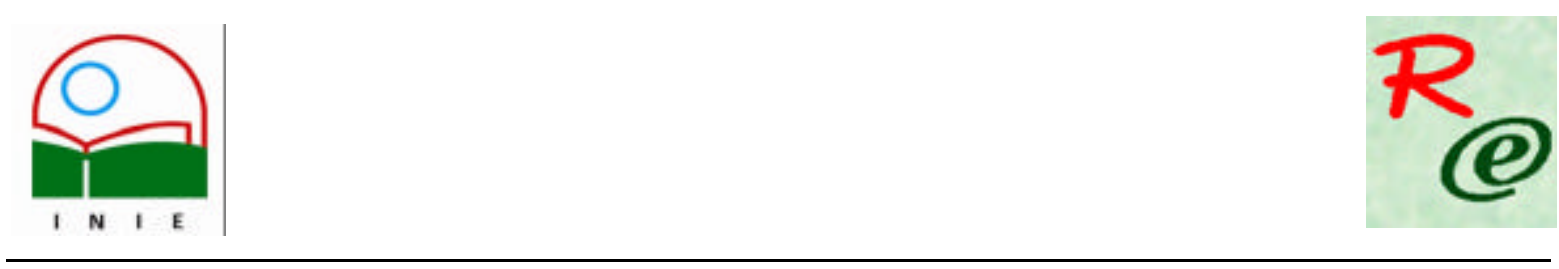

Actualidades Investigativas en Educación

Revista Electrónica publicada por el

Instituto de Investigación en Educación

Universidad de Costa Rica

ISSN 1409-4703

http://revista.inie.ucr.ac.cr

COSTA RICA

\title{
ESTUDIO COMPARATIVO DE LA RESOLUCIÓN DE PROBLEMAS EN EL RENDIMIENTO ESTUDIANTIL EN EL CONTENIDO DE ELECTROQUÍMICA
}

COMPARATIVE STUDY OF THE RESOLUTION OF PROBLEMS IN THE STUDENT YIELD ON THE CONTENT OF ELECTROCHEMISTRY

Volumen 9, Número 1

pp. 1-17

Este número se publicó el 30 de abril 2009

Saida Matute Padrón

Léxida Pérez Peña

Lucia Di' Bacco Vera

La revista está indexada en los directorios:

LATINDEX,$\underline{\text { REDALYC }}$ IRESIE, $\underline{\text { CLASE}}$, DIALNET, DOAJ, E-REVIST@S,

La revista está incluida en los sitios:

REDIE, RINACE, OEI, MAESTROTECA, PREAL, HUASCARAN, CLASCO 


\title{
ESTUDIO COMPARATIVO DE LA RESOLUCIÓN DE PROBLEMAS EN EL RENDIMIENTO ESTUDIANTIL EN EL CONTENIDO DE ELECTROQUÍMICA \\ COMPARATIVE STUDY OF THE RESOLUTION OF PROBLEMS IN THE STUDENT YIELD ON THE CONTENT OF ELECTROCHEMISTRY
}

\author{
Saida Matute Padrón ${ }^{1}$ \\ Léxida Pérez Peña ${ }^{2}$ \\ Lucia Di' Bacco Vera ${ }^{3}$
}

\begin{abstract}
Resumen: Este estudio comparó las metodologías de resolución de problemas de: Reif, Durán y García, a través del rendimiento estudiantil en el contenido de electroquímica. La muestra estuvo representada por 73 estudiantes del Primer Año de Ciencias de la Unidad Educativa Nacional "El Eneal" del Municipio Crespo, Estado LaraVenezuela, año escolar 2006-2007. Veinticinco (25) aprendices pertenecen al grupo experimental uno (GE 1 ) y los grupos experimentales dos $\left(G E_{2}\right)$ y tres $\left(G E_{3}\right)$ están conformados por veinticuatro (24) estudiantes cada uno. Antes de la aplicación de las metodologías, se administró una prueba de conocimientos previos a los tres grupos experimentales; determinándose que los grupos son homogéneos. Al finalizar las tres metodologías, se les aplicó a los tres grupos una post-prueba. Los resultados permitieron concluir que no existe diferencia, estadísticamente significativa, entre el rendimiento estudiantil, en términos del promedio de calificaciones, por los tres grupos que recibieron los tratamientos con las tres estrategias de resolución de problemas utilizadas.
\end{abstract}

Palabras claves: RESOLUCIÓN DE PROBLEMAS, ELECTROQUÍMICA, APRENDIZAJE, RENDIMIENTO ESTUDIANTIL.

\begin{abstract}
This study compared the methodologies of resolution of problems: Reif, and García Durán, through student performance on the content of electrochemistry. The sample was represented by 73 students from the First Year of Science Education National Unity "The Eneal" Crespo Municipio Crespo, Estado Lara-Venezuela, 2006-2007 school year. Twenty-five (25) trainees belong to an experimental group (GE1) and two experimental groups (GE2) and three (GE3) are composed of twenty-four (24) students each. Before the application of methodologies, were adm inistered a test of knowledge prior to the three experimental groups, determined that the groups are homogeneous. At the end of the three methodologies were applied to all three groups a post-test.The results allowed concluding that statistically significant difference between the student yields in terms of the average of qualifications, obtained does not exist in the post-test by the three groups that received training with the three strategies of resolution of used problems.
\end{abstract}

Keywords: RESOLUTION OF PROBLEMS, ELECTROCHEMISTRY, LEARNING, STUDENT YIELD.

\footnotetext{
Thvestigadora de la Universidad Centroccidental Lisandro Alvarado. Dirección electrónica:smatute@ucla.edu.ve

${ }^{2}$ Investigadora de la Unidad Educativa el Eneal. Dirección electrónica: lesidapgjagbto@hotmail.com

${ }^{3}$ Investigador de la Universidad Pedagógica de Barquisimeto. Dirección electrónica:mscldbaccc@hotmail.com
}

Artículo recibido: 17 de septiembre, 2008

Aprobado: 2 de abril, 2009 


\section{Introducción}

El aprendizaje de la ciencia, y en especial la química, se ha caracterizado por un enfoque tradicional de sus métodos, poca relevancia de los contenidos, ausencia de transferencias de conocimientos previos y la falta de actitud positiva hacia la asignatura tal como lo reseña el Centro Nacional para el Mejoramiento de la enseñanza de la Ciencia (CENAMEC, 1999).

En la búsqueda de una mejor enseñanza de la química en el nivel de la Educación Básica de la República Bolivariana de Venezuela, se requiere que el aprendiz desarrolle actitudes y valores que le permitan en cualquier momento transferirlas a una situación para resolver problemas.

Bajo esta misma óptica, el Ministerio de Educación, en el programa de química de noveno grado (2000), sugiere aplicar nuevas estrategias pedagógicas con el fin de que el estudiante adquiera habilidades para el procesamiento de información de manera lógica y aplicarlas a situaciones de la vida diaria.

Sin embargo, por experiencias de las autoras la realidad que se tiene en la Unidad Educativa Nacional "El Eneal", del Municipio Crespo Estado Lara-Venezuela, es algo diferente a lo esperado, porque se observa poca motivación de los alumnos por asignaturas de ciencias, tal es el caso de química como una de las menos aceptadas, bajo rendimiento estudiantil, poca realización de prácticas de laboratorio y un enfoque tradicionalista por parte del docente, lo cual concuerda con lo que señala Rodríguez (1996), que el empleo de estrategias tradicionales, por parte de los educadores, produce en el estudiante deficiencias en su rendimiento estudiantil.

De lo expuesto anteriormente, el CENAMEC y la Oficina de Planificación del Sector Universitario (citados en Lara, 2002) afirman que la educación científica en Venezuela carece de calidad y equidad, lo que se evidencia en los altos índices de deserción escolar, poca creatividad, carencias de estrategias de aprendizaje y el bajo rendimiento estudiantil.

En ese orden de ideas, Ramos (1999) expresa que el CENAMEC, en muchas ocasiones, ha señalado que las dificultades de los educandos al resolver problemas de química se deben a la falta de estrategias metodológicas por parte de los educadores que orienten su resolución. Por esta razón, el mencionado organismo sugiere a profesores (as) que imparten esta asignatura utilizar un enfoque metodológico, organizado y lógico que permita al educando la comprensión y el análisis de los problemas que se les presenten. 
Así mismo, es necesario acotar que el aprendiz de educación Básica, Media Diversificada y Profesional presenta dificultades en la aplicación de algunos conceptos químicos, tal como el tema de electroquímica. Vasini y Donati (2001) señalan que, generalmente, educandos y educadores afirman que las dificultades en dicho contenido se presentan en la identificación del marco conceptual y físico común entre celdas electrolíticas y galvánicas, así como las asociadas a las propias reacciones de óxido-reducción, con los responsables del transporte de la corriente eléctrica a través de la solución y del puente salino o con la interpretación de las cargas asignadas a cada electrodo.

También, Aldaz, Brillas, López, Pingarrón, Ruedas, Fernández y Ruiz (2005) afirman que la gran problemática, por la que atraviesan los aprendices en este contenido, se debe a los complejos desarrollos matemáticos en los que hacen énfasis la mayoría de los educadores, sin dar a conocer la importancia y utilidad de la electroquímica a nivel industrial, ambiental y social, entre otros.

En ese sentido, Colmenares (2006) expresa que existe entre los investigadores de esta área un acuerdo en considerar problemas a todas aquellas situaciones que se plantean a los educandos para las cuales no tienen una solución o un método inmediato para resolverlos.

Lo expuesto indica que está en manos de los profesionales de la educación ayudar al educando en el logro de los objetivos programáticos, para lo cual es necesario convertir el proceso educativo en un sistema creativo, tomando en cuenta la inmensa cantidad de estrategias innovadoras que se ofrecen en la actualidad para llevar a cabo dicho proceso de una manera más eficaz.

En atención a lo planteado anteriormente y tomando en consideración los estudios realizados con anterioridad, en torno a metodologías de resolución de problemas, en este estudio se utilizaron las propuestas por Reif, Durán y García, puesto que se ha demostrado que con el uso de las mismas, los estudiantes incrementan la capacidad de análisis y síntesis y, estadísticamente, se ha comprobado su eficacia en el proceso de resolución de problemas de química y los efectos positivos sobre el rendimiento estudiantil.

\section{Objetivo General de la Investigación}

Medir y comparar la efectividad en el aprendizaje del contenido de electroquímica, mediante la contrastación del rendimiento estudiantil en función del promedio de las calificaciones en las post-pruebas obtenidos por los estudiantes de los grupos experimentales en cada uno de los tratamientos. 


\section{Hipótesis de la Investigación}

$\mathrm{H}_{0}$. No existe diferencia, estadísticamente significativa, en el rendimiento estudiantil en términos del promedio de calificaciones obtenidos en la postprueba, por los estudiantes que fueron tratados con las estrategias de resolución de problemas: Reif, Durán y García.

$H_{a}$ Existe diferencia, estadísticamente significativa, en el rendimiento estudiantil en términos del promedio de calificaciones obtenidos en la postprueba, por los estudiantes que fueron tratados con las estrategias de resolución de problemas: Reif, Durán y García.

\section{Fundamentación Teórica}

Hoy por hoy la resolución de problemas ha adquirido un estatus complejo que va mucho más allá de la tradición como instrumento esencial de evaluación, motivación y aplicación de lo aprendido, hacia ser el centro de propuestas curriculares de cambio cultural en el aprendizaje de las ciencias, entorno a procesos centrados en transformaciones conceptuales y, en particular, procedimentales y actitudinales.

Dos disciplinas, últimamente, han venido a fortalecer la importancia de la resolución de problemas en los procesos de formación científica de las sociedades, estas son la Epistemología y la Didáctica, pues reconocen que el objetivo de las Ciencias no es otro que resolver problemas y que la enseñanza de las Ciencias debe tener como fundamento: la formación de actitudes científicas mediante procesos de investigación en el aula.

Como aplicación de esta idea, buena parte de las nuevas propuestas curriculares en el contexto iberoamericano se han organizado entorno a la resolución de problemas o incluyéndolo como contenido obligatorio, con el fin de permitir a los aprendices desarrollar su metodología y técnicas de investigación y así desempeñarse mejor en la resolución de problemas de la cotidianidad. (Postigo y Gómez, 2005). Por tanto, la enseñanza de la resolución de problemas se vincula con la realidad cotidiana del estudiantado, ocupando el lugar intermedio entre el pensamiento cotidiano y el pensamiento del mundo de la ciencia (Pozo, 2004).

Así mismo, cabe destacar que en la República Bolivariana de Venezuela, con la implantación de los Liceos Bolivarianos, se propone entre uno de sus objetivos la formación del adolescente y joven en la resolución de problemas por investigación, que le son propios y los de su comunidad de manera corresponsable y solidaria. Lo que se pretende con este objetivo es que la resolución de problemas por investigación no sólo pretende dotar al Volumen 9, Número 1, Año 2009, ISSN 1409-4703 
individuo de unos conocimientos fundamentales, desde el punto de vista epistemológico y social mediante el redescubrimiento de los mismos, sino que también y, fundamentalmente, intenta que el educando adquiera unos códigos ordenados de conducta, unos esquemas de comportamiento suficientes para poder desenvolverse en cualquier situación normal de la vida diaria" (Contreras, 2007).

En concordancia con lo anterior, el acercamiento a los procesos de indagación científica, mediante la resolución de problemas, con el consiguiente desarrollo de habilidades básicas, procedimentales e investigativas, puede constituirse en una alternativa eficaz para incrementar la creatividad y la capacidad crítica de los aprendices, lo cual se constituye, a su vez, en fundamento de la autonomía y responsabilidad social.

En concordancia con lo anterior, Novak (Citado por Solaz y López, 2008) considera que la resolución de un problema implica la reorganización de la información almacenada en la estructura cognoscitiva de la persona que lo resuelve, es decir, que hay aprendizaje significativo. En sentido similar, plantea que habrá aprendizaje significativo cuando el material aprendido encaja en la red cognoscitiva ya existente en quien aprende cuando probablemente el trabajo se realiza en la denominada zona de interés óptimo, que quizás corresponde al umbral de dificultad. Esta zona sería intermedia entre la de información conocida y la de desconocimiento total; por consiguiente, constituye un límite personal.

En esos términos, la resolución de problemas podría ser considerada como un proceso mediante el cual una persona que se enfrenta a un problema, trata de identificarlo, de delimitarlo, de explorar posibilidades de resolverlo, de elegir las estrategias adecuadas para lograrlo a partir de sus desarrollos individuales, de llevarlas a la práctica mediante la aplicación de métodos y técnicas apropiados.

Cuando se plantea una situación problemática hay un aspecto que se muestra como punto de partida: el enunciado, la descripción del fenómeno sobre el que se pretende trabajar. Aparentemente, el tipo de situaciones que se pueden plantear y la forma de hacerlo es muy limitado, lo que lleva a cierta uniformidad en la presentación escrita de ejercicios, problemas y prácticas.

Pero esta presentación tiene detrás muchas intenciones que diferencian las distintas formas de hacer este tipo de "tarea docente". La resolución que se dé depende del "modelo didáctico" por el que se opte, emergiendo las diferentes concepciones de la ciencia, de la escuela y del mundo real que tienen los educadores y aprendices. Es decir, aparecen diversas formas de ver una misma situación. 
En concordancia con lo anterior, se puede decir que la resolución de problemas implica una situación de transferencia de conocimientos, ocupando un lugar relevante en el proceso educativo como estrategia de enseñanza, como actividad de aprendizaje y como instrumento de evaluación. En el contexto de enseñanza de la química, son considerados como problemas todos aquellos a los que el estudiante se enfrenta, tanto en situaciones de aprendizaje como de evaluación: problemas propuestos oralmente en el aula, problemas de lápiz y papel enunciados en guías de estudio, problemas experimentales abordados en las clases de laboratorio, pequeñas investigaciones desarrolladas como trabajos especiales, entre otros.

De todo lo antes expuesto, hoy en día existen metodologías de resolución de problemas que ayudan al estudiantado a obtener solución de la situación, tal como se observa a continuación:

\subsection{Modelo de resolución de problemas de Reif}

Reif (citado por Martínez, 2006) señala que la mayor dificultad que presentan los participantes al resolver cualquier tipo de problema es la falta de comprensión del mismo y conocimientos básicos para la resolución. De igual modo, este autor afirma que el triunfo obtenido por expertos en dicho proceso se debe tanto al cúmulo de conocimientos adquiridos como al uso de estrategias.

En este sentido, Reif presenta una serie de procedimientos generales de resolución de problemas que dividen el proceso en tres etapas las cuales son:

- Descripción y análisis

- Descripción Básica

○ Datos

○ Incógnitas

- Relación entre los datos y la incógnita

- Descripción Teórica

- Síntesis de una solución

- Revisión y mejoramiento de la solución.

En la primera etapa se realiza una descripción básica del problema; es decir, se señala la información especificada en el mismo que corresponde a los datos e incógnitas con las respectivas unidades que permitan determinar la relación entre estos dos pasos. Es una Volumen 9, Número 1, Año 2009, ISSN 1409-4703 
descripción y análisis del problema para darle una solución. Además, se recomienda, en esta etapa, redescribir el problema sobre la base de los conceptos necesarios, proporcionados por el conocimiento básico que se tenga sobre el contenido estudiado con el fin de facilitar la búsqueda de la solución.

En la etapa de reconstrucción de la síntesis de una solución, se recomienda el uso de estrategia de refinamientos progresivos, porque la misma permite tomar decisiones principales sin distractores, las cuales pueden generar dudas como guías para tomar otras decisiones, hasta considerar todos los aspectos tratados y obtener la solución del problema.

En la revisión y mejoramiento de la solución, es necesario realizar un chequeo, para determinar si la respuesta obtenida es correcta y razonable y, de esta forma, realizar las mejoras en cuanto a la solución de la situación.

\subsection{Modelo de Resolución de Problemas de Durán a partir de Polya}

A partir de la Metodología de Polya (1996) esta autora diseñó una regla nemotécnica titulada AGUA, y consta de cuatro etapas, en la que el enunciado de cada fase se inicia con cada una de las letras del título de dicha regla. Asimismo, la autora, en cada una de las etapas, sugiere una serie de reglas que los aprendices deben llevar a cabo para la resolución del problema.

Como primera fase, la regla nemotécnica AGUA de Durán establece que se debe: "Abordar la situación problemática", que se lleva a cabo al leer y comprender bien el problema, en esta etapa es necesario conocer los datos disponibles, las incógnitas planteadas y las restricciones existentes en el mismo.

En la segunda etapa se debe: "Generar un plan de acción", en tal sentido, es importante conocer el tema referente al problema para así poder ubicar las fórmulas y ecuaciones aplicables. Seguidamente, se deben asociar las incógnitas con los datos, fórmulas y ecuaciones.

Luego, se procede a nombrar los pasos necesarios para lograr obtener la incógnita, es decir, se deben especificar los cálculos a realizar en cada fórmula, despejar, si es necesario, y ordenar las operaciones que se vayan a emplear.

La tercera etapa consiste en: "Usar el plan de acción", allí se deben seguir paso a paso las operaciones planificadas en la etapa anterior, es decir, realizar despejes, transformar unidades, introducir datos con sus respectivas unidades en las fórmulas correspondientes, para así obtener la respuesta a las incógnitas planteadas. 
La cuarta etapa hace alusión al: "Análisis de los resultados" obtenidos, para lo que se debe seguir, en detalle, cada uno de los procedimientos llevados a cabo anteriormente y de esta forma, evaluar dichas respuestas y así proponer otras posibles vías de solución.

Esta estrategia o regla nemotécnica según Durán (1996) la resume así:

Abordar la situación problemática

"PEDIR"

Generar un plan de acción

"CUANDO"

Usar el plan de acción

"SIENTA"

Analizar los resultados

"SED"

\subsection{Modelo de resolución de problemas de García}

La metodología de García comprende las siguientes etapas para la resolución de problemas cuantitativos:

Representación y replanteamiento del problema: Según García (2003), en esta etapa el estudiante debe elaborar un modelo del problema y traducir la información escrita en el mismo a un sistema sobre el cual se pueda operar a través de las siguientes herramientas:

1. Leer minuciosamente el problema.

2. Construir un esquema a manera de gráfica para crear una imagen clara de la situación física a la cual corresponde el problema.

3. Tratar de definir cuál es el objetivo del problema, preguntándose ¿Qué es lo que se pide en el problema?

4. Hacer una lista de los datos y de las incógnitas que presenta el problema.

5. Colocar los datos en el esquema y debajo de cada uno colocar sus respectivas unidades.

6. Buscar alguna relación entre las incógnitas y los datos, tratando de relacionar las cantidades conocidas con los valores desconocidos.

7. Escribir en el lenguaje propio las relaciones claves encontradas.

Presolución: Esta etapa consiste en presentar la información necesaria para la resolución del problema y hacer una estimación del procedimiento a seguir y de los posibles resultados, las herramientas que se deben manejar son:

1. Seleccionar y escribir la información que considere importante para la resolución del problema. 
2. Enumerar las ecuaciones matemáticas o principios físicos relacionados con las cantidades presentadas en el problema.

3. Hacer una estimación de la respuesta, ordenando las magnitudes y usando las ecuaciones probables y asignarles valores razonables, con el fin de obtener un número aproximado como respuesta.

4. Si el problema está muy largo, o complicado dividirlo, en subproblemas más pequeños y luego solucionarlo por partes.

Resolución y revisión: Se refiere a los procesos de transformación de los datos y de las incógnitas, además, incluye la ejecución de cálculos pertinentes para obtener las respuestas requeridas y la revisión de la misma, utilizando las siguientes herramientas:

1. Transformar los datos que se consideren necesarios, mediante los factores conversión.

2. Sustituir datos en fórmulas y ecuaciones que permitan establecer el valor de las incógnitas.

3. Efectuar los cálculos necesarios.

4. Verificar si el valor de la respuesta es razonable o posible, es decir, si tiene o no sentido.

5. Comprobar que la respuesta cumpla con las condiciones impuestas en el enunciado del problema.

6. Revisar si existen otros caminos de resolución que lleven a la misma respuesta.

7. Realizar una reflexión global acerca de lo que aportó la resolución del problema en lo metodológico y en lo conceptual.

\section{Metodología}

El presente estudio es una investigación de campo con un diseño cuasi-experimental, según Hernández, Fernández y Baptista (2003) los sujetos no son asignados al azar por las autoras en los grupos experimentales, sino que los mismos ya estaba formados antes de la investigación, es decir, son grupos intactos.

La población estuvo representada por ciento cuarenta y ocho (148) alumnos(as) en edades comprendidas entre 16 y 17 años que constituían las seis secciones del primer año de Ciencias de Educación Media Diversificada y Profesional de la Unidad Educativa Nacional El Eneal, ubicada en el Municipio Crespo, Estado Lara-Venezuela, y que cursaron la asignatura química durante el año escolar 2006-2007. 
La muestra en estudio estuvo conformada por setenta y tres (73) educandos cursantes de tres secciones, las cuales fueron seleccionadas al azar, quedando constituido el grupo experimental uno $\left(\mathrm{GE}_{1}\right)$ con veinticinco (25) aprendices y los grupos experimentales dbs $\left(\mathrm{GE}_{2}\right)$ y tres $\left(\mathrm{GE}_{3}\right)$ con veinticuatro (24) estudiantes cada uno.

Los tratamientos aplicados se desarrollaron en cinco (5) semanas en sesiones de seis (6) horas semanales de cuarenta y cinco minutos (45) cada una, durante las cuales se realizaron las mismas actividades de inicio, desarrollo y cierre en las sesiones uno, dos y tres y desde la cuatro (4) hasta la trece (13) variando, únicamente, en la metodología de resolución de problemas de Reif $\left(\mathrm{GE}_{1}\right)$ sección $\mathrm{E}$, Durán $\left(\mathrm{GE}_{2}\right)$ sección $B$ y García $\left(\mathrm{GE}_{3}\right)$ sección D.

Las actividades de inicio, fundamentalmente, se efectuaron para diagnosticar las concepciones previas de los participantes a través de la elaboración de rompecabezas y sopas de letras, a fin de propiciar el aprendizaje del tema Electroquímica en los contenidos conceptuales, procedimentales y actitudinales.

En el desarrollo de los aspectos teóricos, se utilizaron materiales de apoyo como ideogramas, mapas conceptuales, flujogramas, material mimeografiado y materiales de uso común en la vida diaria para las demostraciones, entre otros, y para explicar los tópicos referidos a la resolución de problemas se utilizó el pizarrón, tiza, borrador y guías de situaciones problemáticas.

Las actividades de cierre se realizaron con el propósito de aclarar las dudas en torno al objetivo desarrollado en cada sesión de clase, estas se efectuaron a través de preguntas elaboradas por ellos y las docentes a través de dinámicas como cruzaletras. Asimismo, en esta fase se reflexionaban acerca de las ideas, conceptos y procedimientos estudiados, los productos obtenidos y las dificultades detectadas en cuanto al proceso de resolución de problemas y otros aspectos.

Por otra parte, la investigación se desarrolló de la siguiente manera: la sesión $N^{\circ} 1$, se inició la clase con la presentación de la información a cada grupo experimental acerca del estudio por realizar y referida a la importancia de la investigación con la aplicación de las tres estrategias de resolución de problemas. Seguidamente, se procedió a la aplicación de la Prueba de conocimientos previos (PCP) en las secciones E, B y D. Por último, se les informó a los aprendices sobre la actividad a llevar a cabo en la próxima sesión de clase según los resultados de la prueba. 
La sesión $N^{\circ} 2$ se inició informando a los estudiantes sobre los resultados obtenidos en la prueba de conocimientos previos. Luego, se realizó una dinámica de inicio a través de la elaboración de una sopa de letras, con conceptos claves sobre los contenidos que se desarrollarían durante el transcurso del trabajo de investigación.

Más adelante, se presentó, a través de un ideograma, el contenido por desarrollar, se explicaron los conceptos de electroquímica, reacciones redox, celda electroquímica, clasificación de las celdas, electrodo, electrolito, corriente eléctrica y electrólisis, mediante el uso de mapas de conceptos.

Además, se les proporcionó, a los participantes, materiales impresos sobre resúmenes de investigaciones realizadas en torno al tema desarrollado, esto es con el fin de valorar la importancia de los procesos electroquímicos allí señalados. Para finalizar, se aclararon las dudas sobre cada aspecto desarrollado en la sesión de clase y se dieron instrucciones para la realización de actividades con la información presentada con anterioridad.

En la sesión $N^{\circ} 3$, los educandos realizaron exposiciones con el propósito de demostrar el carácter conductor de la corriente eléctrica por sustancias presentes en las frutas.

La sesión $N^{\circ} 4$, se inició agrupando a los aprendices por parejas y suministrándoles, a cada uno, un sobre que contenía palabras recortadas en pequeños trozos para armar un rompecabezas; esto se hace con el fin formar palabras y cada palabra es definida por cada grupo. Luego, se solicitó la colaboración de un alumno para leer el objetivo de la clase escrito en una lámina. Seguidamente, se procedió a explicar el enunciado de la primera y segunda Ley de Faraday a través de un mapa conceptual.

A partir de la sesión $N^{\circ} 5$ hasta la 11 , se resolvieron, a través de la metodología de Reif, Durán y García problemas donde se utilizaban las relaciones estequiométricas de las reacciones que ocurren en las celdas electroquímicas, la constante de Faraday, así como la intensidad de corriente eléctrica. En esta fase, los alumnos seleccionados en grupos, así como individualmente, realizaban una serie de problemas aplicando los conocimientos adquiridos al inicio en clase haciendo énfasis en la metodología correspondiente para cada grupo en estudio.

De igual manera, en la sesión $N^{\circ} 12$ y 13 se utilizaron las metodologías de resolución de problemas de Reif, Durán y García para balancear ecuaciones redox por el método de cambio del número de oxidación y por el método del ión electrón. Para desarrollar el contenido, se les proporcionó a los colaboradores material mimeografiado sobre las reglas Volumen 9, Número 1, Año 2009, ISSN 1409-4703 
para determinar el número de oxidación de un elemento, los pasos para balancear ecuaciones por el método del cambio del número de oxidación y del ión electrón, y se les explicó dicho contenido a través del uso de la metodología especificada para cada sección experimental.

Cabe destacar, que al finalizar cada sesión se resolvieron los problemas. De igual manera, se les asignaban a los educandos actividades para realizar en el hogar, con el fin de afianzar los conocimientos adquiridos, mediante el uso de la metodología de resolución de problemas de Reif, Durán y García según sea el caso. Asimismo, dichos problemas eran evaluados, posteriormente, al iniciar cada sesión aclarándose dudas que presentaban los aprendices con respecto al contenido de química, como también al referido a la metodología utilizada.

\section{Resultados y Discusión}

Los resultados obtenidos en la investigación se presentan, en primer lugar, al comparar los resultados de la aplicación de la prueba de conocimientos previos (PCP) y, en segundo lugar, los resultados obtenidos en la post-prueba.

El coeficiente estandarizado de asimetría se aplicó sobre los resultados de la prueba de conocimientos previos, aplicada a los tres grupos experimentales, para verificar si los grupos en estudio presentaban un comportamiento estadísticamente normal. Los valores tomados como referencia para una distribución normal están comprendidos entre los límites 2 a +2 , según la hoja estadística computarizada Microsoft Excel 97. Los resultados se muestran en el cuadro 1.

\section{Cuadro 1}

Coeficiente Estandarizado de Asimetría

Estadígrafo
Coeficiente Estandarizado de
Asimetría

Nota: $\mathrm{GE}_{1}, \mathrm{GE}_{2}$ y $\mathrm{GE}_{3}=$ grupos experimentäles.

Los resultados de la prueba, obtenidos por los tres grupos, se encuentran entre los límites -2 y +2 , con lo cual se concluyó que los gupos tienen una distribución de frecuencia aceptable como normal. 
De igual forma, a los resultados de esta prueba, se le aplicó la prueba de homogeneidad de la varianza de Levene (Hernández, González, 2007), con el propósito de verificar si los grupos en estudio eran homogéneos para ser comparados. Los resultados de la prueba se presentan en el cuadro 2.

Cuadro 2

Prueba de homogeneidad de la varianza de Levene

\begin{tabular}{|c|c|c|c|}
\hline Estadístico de Levene & Gl 1 & gl 2 & Sig \\
\hline 2,168 & 2 & 63 & 0,123 \\
\hline
\end{tabular}

Nota: gl = grados de libertad; Sig = niveì de significancia experimentāi

Se puede observar que el nivel de significancia experimental es de 0,123 ; lo que significa que es mayor que el nivel de significancia fijado de antemano $(0,05)$. Por tal razón, se interpreta que no existe diferencia significativa en las varianzas de los grupos en cuanto a conocimientos previos y, por ende, se concluye que los grupos pueden ser comparados.

\section{Cuadro 3}

Análisis de Varianzas (ANOVA)

\begin{tabular}{|c|c|c|c|c|c|}
\hline$\overline{F V}$ & SC & $\mathrm{gl}$ & $\mathrm{MC}$ & $\mathbf{F}$ & Sigig \\
\hline Inter.-grupos & 8,471 & 2 & 4,235 & 0,800 & 0,454 \\
\hline Intra-grupos & 333,347 & 63 & 5,291 & & \\
\hline Total & 341,818 & 65 & & & \\
\hline
\end{tabular}

Nota: FV= fuente de variabilidad; $\mathrm{SC}=$ suma de cuadrados; gl = grados de libertad; $\bar{M} \mathrm{C}=$ media cuadrática; F= F práctico o experimental; Sig = nivel de significancia.

Según se observa en el cuadro 3 , el nivel de significancia experimental $(0,454)$ obtenido a través del análisis de varianza, es mayor que el nivel de significancia fijado de antemano $(0,05)$, por lo tanto, se interpreta que no existe diferencia estadísticamente significativa entre el rendimiento estudiantil, en términos del promedio de las calificaciones obtenidos por los tres grupos en estudio, lo que significa que éstos poseen condiciones similares; es decir, son equivalentes en cuanto a la media de los conocimientos previos. 
Una vez concluido el proceso de aplicación simultánea de la Post-prueba, se llevó a cabo el análisis de Varianza (ANOVA), para determinar cuál de las tres estrategias de resolución de problemas produjo efecto significativo sobre el rendimiento estudiantil, en términos del promedio de calificaciones en el aprendizaje del contenido de electroquímica. Los resultados de dicho análisis se presentan en el siguiente cuadro.

\section{Cuadro 4}

Análisis de Varianzas (ANOVA) de la Post-prueba

\begin{tabular}{|c|c|c|c|c|c|}
\hline$\overline{F V}$ & $\mathrm{SC}$ & $\mathrm{gl}$ & $\mathrm{MC}$ & $\mathbf{F}$ & Sìg \\
\hline Īnter.-grupos & 77,779 & 2 & 38,890 & 2,958 & 0,060 \\
\hline Intra-grupos & 762,581 & 58 & 13,148 & & \\
\hline Total & 840,361 & 60 & & & \\
\hline
\end{tabular}

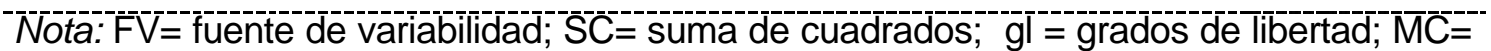
media cuadrática; F= Fpráctico o experimental; $\mathrm{Sig}=$ nivel de significancia

Como el nivel de significancia experimental $(0,060)$ es mayor que el nivel de significancia fijado de antemano $(0,05)$; representa la aceptación de la hipótesis nula de la investigación, la que estableció que no existe diferencia estadísticamente significativa en el rendimiento estudiantil, en términos de promedio de las calificaciones de los alumnos obtenidos en la post-prueba, para el aprendizaje de de electroquímica, por los grupos de alumnos tratados con las estrategias de resolución de problemas: Reif, Durán y García; es decir, las mismas son igualmente efectivas.

De lo antes expuesto, expresa Ramos (1999), que el CENAMEC ha señalado que las dificultades que presentan los educandos al resolver problemas de química, se deben a la falta de estrategias metodológicas por parte de los docentes que orienten su resolución. De hecho, esto se evidencia en dicha investigación porque las estrategias de Reif, Durán y García produjeron un efecto significativo en el aprendizaje de la electroquímica. Tal como lo plantea Novak (Citado por Solaz y López, 2008) que el aprendizaje significativo se produce cuando el material aprendido encaja en la red cognoscitiva ya existente en quien aprende; y eso fue lo que ocurrió con los estudiantes al resolver problemas de electroquímica con las diferentes metodologías presentada en este estudio. 


\section{Conclusiones}

Al considerar los objetivos y resultados obtenidos en el presente estudio, se presentan las siguientes conclusiones:

1. Estadísticamente, se comprobó el comportamiento normal, homogéneo y equivalente de los grupos antes de la aplicación de las metodologías de Reif, Durán y García a través de la prueba de conocimientos previos (PCP).

2. El rendimiento estudiantil, en términos del promedio de calificaciones obtenido en la postprueba en el contenido electroquímica por el grupo experimental tratado con la metodología de Reif, evidencia que no existe diferencia estadísticamente significativa de la misma, con respecto a la metodología de Durán y García.

3. Igualmente, al comparar el grupo instruido con la metodología de Durán, en relación con los tratados con la metodología de Reif y García, se observa que no existe diferencia estadísticamente significativa en el rendimiento estudiantil en términos de los promedios de las calificaciones obtenidos en la post-prueba en el contenido electroquímica del primero con respecto a los otros dos.

4. Asimismo, se evidenció que no existe diferencia estadísticamente significativa en el rendimiento estudiantil en términos del promedio de calificaciones obtenido en la post prueba sobre electroquímica, del grupo de educandos instruidos con la metodología de García y los tratados con la metodología de Reif y Durán.

5. Al comparar estadísticamente el rendimiento estudiantil en términos de los promedios de calificaciones obtenidos en la post-prueba sobre electroquímica en los tres grupos experimentales tratados con las metodologías de resolución de problemas de Reif, Durán y García, se comprobó que las mismas producen efecto favorable sobre el rendimiento estudiantil en la asignatura química de los aprendices del primer año de Ciencias de la Unidad Educativa Nacional "El Eneal".

\section{Recomendaciones}

Con base en los resultados obtenidos en el estudio y las conclusiones planteadas anteriormente, se realizan las siguientes recomendaciones:

1. Usar las metodologías de resolución de problemas de Reif, Durán y García en otros contenidos de química, puesto que se evidenció que cada una posee una serie de ventajas que permiten a los aprendices adquirir habilidades y destrezas en la resolución de situaciones. 
2. Realizar investigaciones con las mismas metodologías en condiciones diferentes de tiempo y ambiente, con el propósito de verificar los resultados de este estudio.

3. Efectuar investigaciones para comparar las metodologías investigadas con otras diferentes a las utilizadas en este estudio y, en otros contenidos y niveles educativos.

\section{Referencias}

Aldaz, Amparo, Brillas, Eduardo, López, Juan, Pingarron, José, Rueda, Mercedes, Fernández, Tatiana y Ruiz, Jesús. (2005). La Enseñanza de la Electroquímica. Recuperado el 2 octubre de 2007, de: http://www.upct.es/electroquím ica/MesaRedondaElectroquímica.pdf.

Centro Nacional para el Mejoramiento de la Enseñanza de la Ciencia. (1999). Carpetas de Ciencias Naturales para Docentes de Educación Básica. Caracas: CENAMEC.

Colmenares, Carlos (2006) Análisis de necesidades en el aprendizaje de resolución de problemas cuantitativos de Química en el Instituto Pedagógico de Barquisimeto. Revista de Investigación y Postgrado, 3 (4), 37-51.

Contreras, Franklin. (2007). La resolución de problema por investigación (Reporte Técnico). Universidad Pedagógica Experimental Libertador, Caracas. Venezuela

Durán, María. (1996). Estrategias de entrenamiento y su efecto sobre las habilidades en resolución de problemas de química. Trabajo de grado de Maestría en Educación. Universidad Pedagógica Experimental Libertador, Instituto Pedagógico Luís Beltrán Prieto Figueroa, Barquisimeto.

García, José. (2003). Didáctica de las ciencias. Resolución de problemas y desarrollo de la creatividad. Colombia: Magisterio.

García, Mena. (1994). La solución de problemas, como estrategia para el desarrollo de la actividad cognoscitiva en el aprendiz. Una revisión bibliográfica. Revista del Departamento de Prácticas Docentes. 6 (4), 95-123.

Hernández, Sampieri, Fernández, Collado y Baptista, Lucio. (2003). Metodología de la Investigación. México: Mc Graw- Hill.

Hernández, René y González, Mónica. (2007). Estadística con SPSS y Metodología de la Investigación. México: trillas.

Lara, José. (2002). Determinación de una estrategia para medir la capacidad de resolver problemas de los alumnos de un curso introductorio de Física (Reporte Técnico). CENAMEC, Caracas. Venezuela.

Martínez, Nelsón (2006). Efecto de la estrategia de enseñanza problemática en el aprendizaje de las celdas galvánicas. Trabajo de grado de Maestría no publicado. 
Universidad Pedagógica Experimental Libertador, Instituto Pedagógico Luis Beltrán Prieto Figueroa. Barquisimeto-Venezuela.

Ministerio de Educación (2000). Oficina Sectorial de Planificación y Presupuesto. Programa de articulación del nivel de Educación Media Diversificada y Profesional, asignatura química primer año. Caracas: Autor.

Palacios, Carmen y Zambrano, Encarnación. (1993). Aprender y enseñar ciencia: una relación a tener en cuenta. Recuperado el 31 de agosto 2006, de http://www.bnm.me.gov.ar/cgi-

bin/wxis.exe/opac/? IsisScript=opac/opac. $x$ is\&dbn=CEDOC\&tb=aut\&src=link\&query=PA LACIOS,\%20CARLOS\&cantidad=\&formato $=\&$ sala $=$.

Polya, George. (1996). Cómo plantear y resolver problemas. Editorial Trillas. México.

Postigo, Eduardo y Gómez, José. (2005). Fundamentos de un método de enseñanza en la resolución de problemas. Revista de Educación. Número 282. Pág 151-160.

Pozo, Ignacio. (2000). Aprendizaje de Estrategias para la Solución de Problemas en Ciencia. Alambique. № 5 Año 2. Julio. Pág 16 - 26. Didáctica de las Ciencias Experimentales. Barcelona: Graó.

Ramos, Oscar. (1999). Desarrollo de habilidades intelectuales a través del uso de estrategias innovadoras en la resolución de problemas de química en educación básica. Trabajo de grado de Maestría en Educación. Universidad Pedagógica Experimental Libertador, Instituto Pedagógico de Maracay, Venezuela.

Solaz, Joan y López, Vicente. (2008). Conocimiento previo, modelos mentales y resolución de problemas. Un estudio con alumnos de bachillerato. Revista Electrónica de Investigación Educativa. 10 (1). Recuperado el 8 de diciembre de 2008, de: http://redie.uabc.mx/vol10no1/contenido-solaz.html

Vasini, Enrrique y Donati, Edgardo. (2001). Uso de analogías adecuadas como recurso didáctico para la comprensión de los fenómenos electroquímicos en el nivel universitario inicial. Recuperado el 3 de diciembre de 2006, de http://www.bib.uab.es/pub/enseñazadelasciencias/ 